Bundesgesundheitsbl 2020 - 63:541-547 https://doi.org/10.1007/s00103-020-03125-8 Online publiziert: 3. April 2020

(c) Der/die Autor(en) 2020

\title{
Norbert Schmacke ${ }^{1,2}$
}

'Institut für Public Health und Pflegeforschung, Universität Bremen, Bremen, Deutschland

${ }^{2}$ Bremen, Deutschland

\section{Homöopathie: Heilslehre „ohne Substanz"}

Homöopathen als essenziell betrachtet werden:

„Similia similibius curentur“ („Ähnliches möge durch Ähnliches geheilt werden“) bedeutet, dass wenn ein verabreichter Stoff typische Krankheitssymptome bei Gesunden auslöst, ein Medikament gegen eine von solchen Symptomen gekennzeichnete Krankheit gefunden ist. Anders gesagt: Krankheiten können geheilt werden, wenn die im Versuch bei Gesunden eingesetzten Medikamente dieselben oder ähnliche Beschwerden auslösen, wie sie bei Kranken zu finden sind.

Hahnemann meinte diese Idee auf einen Selbstversuch stützen zu können, bei dem er die damals gegen Malaria eingesetzte Chinarinde genommen hatte. Er schrieb dazu [2]: „Die Füße, die Fingerspitzen, u. s. w. wurden mir erst kalt, ich ward matt und schläfrig, dann fing mir das Herz an zu klopfen, mein Puls ward hart und geschwind, eine unleidliche Ängstlichkeit, ein Zittern (aber ohne Schauder), eine Abgeschlagenheit durch alle Glieder; dann ein Klopfen im Kopfe, Röthe der Wangen, Durst, kurz alle mir sonst beim Wechselfieber gewöhnlichen Symptome erschienen nacheinander; doch ohne eigentlichen Fieberschauder." Er meinte also, durch die Einnahme der Chinarinde bei sich Malariasymptome hervorgerufen zu haben - der Prototyp eines homöopathischen Arzneimittelbildes, zu dem das systematische Aufschreiben von geschilderten Empfindungen gesunder Testpersonen gehört.

Immer wieder wurde versucht, diesen von Hahnemann als sicher angenommenen Zusammenhang zwischen Chinarinde und Fiebererzeugung zu reproduzie- ren: vergebens. Es entstand, wie auch bei anderen getesteten Substanzen, ein inkohärentes Sammelsurium von Symptomen, die im „Repertorium“, dem Nachschlagewerk der Homöopathie, zusammengefasst wurden. Aus diesem können Homöopathinnen und Homöopathen „das richtige Medikament“ heraussuchen. Es sei am Rande angemerkt, dass in einem starken Kontrast zu dieser Individualisierungsannahme das häufige $\mathrm{Zu}$ rückgreifen auf wenige „bewährte“ Präparate in der Homöopathie steht, ein Schematismus, der es auch ermöglicht, dass Laien sich, ihre Angehörigen und ihre Tiere über eine „Hausapotheke“ weitgehend autark versorgen.

Am Beginn der Homöopathie steht mit dem Chinarindeexperiment also eine manifeste Selbsttäuschung, eine nach wissenschaftlichen Kriterien nicht nachvollziehbare Beobachtungsdeutung des Stifters der Theorie. Es ist mehr als erstaunlich, dass Generationen von Homöopathinnen und Homöopathen zu keinem Zeitpunkt durch diesen leicht erkennbaren Irrtum irritiert worden sind.

Das zweite Grundprinzip der Homöopathie ist das „Verdünnungsprinzip“: Die Wirkstoffe werden bis zum Fehlen der Nachweisbarkeit der Ausgangssubstanz stufenweise hochgradig verdünnt und potenzieren damit ihre klinische Wirksamkeit. Als Heilmittel in der Homöopathie gilt fast alles: Pflanzen, Mineralstoffe, Tiere, Schleim, Kot. Die Homöopathie ist - das kann hieraus abgeleitet werden - entgegen der Annahme vieler Menschen - insofern auch keine „Naturheilkunde“. Das fertige Produkt enthält im Konsens mit der homöopathischen Lehre von einer bestimmten 
Verdünnung ab keine Moleküle mehr: Die im Handel erhältlichen Globuli der "Hochpotenzen" bestehen nur noch aus Milchzucker. Homöopathen sind aber davon überzeugt, dass der Geist der Ausgangsprodukte durch das hochgradige Verdünnen und Verschütteln wirksam erhalten bleibt (sogenanntes Wassergedächtnis) und sogar erst dadurch seine hohe, gewünschte immaterielle Wirksamkeit entfaltet.

Das Lehrgebäude der Homöopathie fußt somit auf willkürlichen und nur obskur zu nennenden Annahmen, die außerhalb aller wissenschaftlichen Grundprinzipien und des gesunden Menschenverstandes angesiedelt sind, auf die wir unser heutiges Leben in den Natur- und Geisteswissenschaften stützen. Oft wird von Homöopathen geltend gemacht, dass sie sich ja doch auf wiederholte Beobachtungen stützen und dies sei doch wissenschaftlich legitim. Es fehlen in der Homöopathielehre allerdings jegliche Ansätze der Nachvollziehbarkeit ihrer Krankheitsdeutungen - es bleibt bei hoch variablen individuellen Einzelbeobachtungen, deren Evidenz nach den international üblichen Standards der evidenzbasierten Medizin Wirksamkeit nicht belegen kann.

Die letzte Ausweichreaktion der Homöopathen ist der Verweis auf heute noch nicht erklärbare Wirkprinzipien. Quantentheorie und Nanotechnologie werden als Hoffnungsträger bemüht, ohne dass es hierfür Belege aus der Physik geben könnte. Die Homöopathie ist mit derartigen Spekulationen nicht allein. Der Neurowissenschaftler Bruce Hood hat in SuperSense. Why we Believe in the unbelievable die Vielfältigkeit von Glaubenskonzepten dargestellt, ihre Bedeutung für eine wie auch immer trügerische Sinnstiftung analysiert und die naheliegende Schlussfolgerung gezogen, dass auf Glauben basierende Heilslehren nicht durch wissenschaftliche Beweisführungen erschütterbar sind [3]. Das leitet unmittelbar über zur Debatte, ob es wissenschaftliche Nutzenbelege für diese von einer stattlichen Community von Homöopathieanhängern wertgeschätzte Lehre gibt.

\section{Nutzenbelege und Heilungs- versprechen}

Innerhalb der wissenschaftsorientierten Medizin wird nun unterschiedlich eingeschätzt, ob es überhaupt sinnvoll ist, sich mit der Frage des Nutzens der Homöopathie mittels der klassischen klinischen Studien zu beschäftigen. Weymayr [4] hat argumentiert, dass Konzepte, die fundamental gegen wissenschaftliche Gesetze verstoßen, als nicht scientiabel aus der Forschungswelt herauszuhalten sind. Es zeigt sich nun, dass führende Homöopathinnen und Homöopathen seit etwa 20 Jahren die frühere Haltung verlassen haben, die Homöopathie sei so individuell und fundamental anders als die „Schulmedizin“ (ein Kampfbegriff der Homöopathen aus dem 19. Jahrhundert [5], der in der nationalsozialistischen Diktion u.a. als ,jüdisch-marxistische" Schulmedizin weitergeführt wurde [6]), dass sie sich den methodischen Grundregeln der naturwissenschaftlichen Medizin nicht unterwerfen könne. Seither argumentieren führende Vertreter der Homöopathie mit klinischen Studien, gar mit dem Vorliegen kontrollierter randomisierter Studien und erzeugen damit in der Öffentlichkeit den Eindruck, sie seien längst in der Welt der Wissenschaft angekommen. Dies gilt beispielsweise für den Einsatz der Homöopathie bei dramatischen Erkrankungen wie Krebs, Schlaganfall oder Blutvergiftung [7]. Ziel dieser neuen Entwicklung ist es, von der akademischen Welt als gleichberechtigt und förderungswürdig anerkannt zu werden.

Im Gegensatz zu Weymayr argumentieren nicht wenige Vertreter der evidenzbasierten Medizin, man könne die in den Zeitschriften der Komplementärmedizin publizierten Homöopathiestudien nicht außer Acht lassen. Es existiert innerhalb der evidenzbasierten Medizin international eine Reihe von Metaanalysen, die aber alle zu demselben Ergebnis gelangen, dass die Homöopathie keine spezifische substanzbezogene Wirkung jenseits von Placeboeffekten hat. Dieser Befund ist relevant, weil die meisten Homöopathen darauf bestehen, dass ihr Behandlungskonzept aufder spezifischen Wirkung der eingesetzten Präparate beruht und eben nicht auf den Placebo- bzw. Kontextfak- toren, die man mit gutem Grund für jede Art von Heilversuch geltend machen kann.

Verwiesen sei bezüglich fehlender Nutzenbelege der Homöopathie auf die Publikation des National Health and Medical Research Council (NHMRC) in Australien [8] und auf den Bericht des Science and Technology Committee des englischen House of Commons [9]. Der englische Bericht enthält über die Evidenzanalyse hinaus zwei zentrale Aussagen: Weitere Forschung zum Nutzen der Homöopathie ist unnötig und der National Health Service solle jegliche Unterstützung der Homöopathie in Forschung, Lehre und Praxis einstellen, damit nicht in der Öffentlichkeit der Eindruck entstünde, es handele sich doch um eine nützliche Therapie.

Homöopathen stellen die genannten Metaanalysen strittig. So hat die wissenschaftliche Gesellschaft für Homöopathie e. V. (WissHom) im Jahr 2016 einen Bericht zum aktuellen Stand der Forschung („Forschungsreader") vorgelegt [10], der zu dem Ergebnis kommt, dass durch methodisch hochwertige klinische Studien die spezifische Wirksamkeit der Homöopathie bewiesen sei. Kernstück des Readers ist eine Metaanalyse von Mathie et al. (2014), die auf der Webseite von WissHom wie folgt kommentiert wird [11]: „Die neueste Metaanalyse von Mathie zeigt auch nach Cochrane Kriterien ein positives Ergebnis für individualisierte Homöopathie. Angewendet unter Alltagsbedingungen in der Praxis (Versorgungsforschung) wird konsistent in den meisten Studien eine Verbesserung von Beschwerden und Lebensqualität durch homöopathische Ärzte berichtet. In der Hälfte aller ökonomischen Analysen werden Kostenersparnisse dokumentiert."

Ohne hier im Einzelnen auf die Unzulänglichkeiten dieser sogenannten Metaanalyse einzugehen (siehe hierzu [12]), sei schlicht auf die Schlussfolgerung der Autorengruppe aus ihrer eigenen Arbeit hingewiesen [13]: „, Medicines prescribed in individualised homeopathy may have small, specific treatment effects ... The low or unclear overall quality of the evidence prompts caution in interpreting the findings. New high-quality RCT research is necessary to enable more decisi- 
ve interpretation." Man muss sich vergegenwärtigen, dass diese Metaanalyse von den Homöopathen als wesentlich eingestuft wird: Niemand in der Welt der evidenzbasierten Medizin würde ein dergestalt hochgradig zurückhaltend bewertetes Verfahren für die Behandlung empfehlen. Es erweist sich im Lichte solch selektiven bis verfälschenden Umgangs mit Publikationen als unmöglich, mit überzeugten Vertretern der Homöopathie über Forschungsergebnisse $\mathrm{zu}$ diskutieren.

Claudia Witt, von 2008 bis 2013 Inhaberin der von der Carstens-Stiftung geförderten Stiftungsprofessur für Forschung in der Komplementärmedizin/ Integrativen Medizin am Institut für Sozialmedizin, Epidemiologie und Gesundheitsökonomie der Charité, zieht in einem Interview die Bilanz, dass mehr als Placeboeffekte durch Homöopathie nicht zu erwarten sind: „Seit über fünf Jahren finden Sie von mir eine offizielle Stellungnahme zur Homöopathie im Internet. Meine Aussage - dass nicht belegt ist, dass homöopathische Arzneimittel mehr als ein Placebo sind - gilt auch heute noch. Die Studienergebnisse zur Wirksamkeit sind uneinheitlich, und meine Einschätzung basiert auf der zumeist schlechten Qualität der Studien. Man kann aber nicht einfach sagen, Homöopathie sei wirkungslos. Erstens ist Homöopathie mehr als die Gabe von Arzneimitteln. Zweitens ist es methodisch nicht korrekt, einfach den Umkehrschluss zu ziehen, im Sinne von: Nun liege der Beweis vor, homöopathische Arzneimittel seien ein Placebo. Das lässt auch die Qualität der Studien nicht zu. Prinzipiell ist dies aber eher eine akademische Diskussion, die wichtige versorgungsrelevante Information ist: Es konnte nicht gezeigt werden, dass homöopathische Arzneimittel besser wirken als Placebo" [14].

Es sei noch auf ein weiteres häufig von Homöopathiebefürwortern vorgetragenes Argument hingewiesen: Die „konventionelle“ Medizin sei ja keineswegs umfassend auf ihre Wirksamkeit hin untersucht, vieles im Alltag der Medizin sei gar unwirksam und schädlich. Es ist das Kernanliegen der evidenzbasierten Medizin, unzureichend untersuchte
Verfahren herauszufiltern. Eine Relativierung innerhalb schlecht oder gar nicht belegter Verfahren ist demgegenüber als nicht statthaft anzusehen.

Viele Menschen nehmen an, dass Homöopathie wirklich nur bei Bagatellerkrankungen und sonst rein additiv zum Einsatz kommt. Es ist aber eine Tatsache, dass Vertreter der Homöopathie auch Schwerstkranken immer wieder Heilung oder substanzielle Besserung durch alleinige oder zusätzliche Gabe von Homöopathika versprechen. Die Argumentationsebene wird hierbei häufig gewechselt: Einerseits wird auf künftige methodisch hochwertige Forschung gesetzt, andererseits wird behauptet, dass relevante Therapieergebnisse bereits vorhanden wären. Es gibt Homöopathen die öffentlich angeben, auch Kranke mit metastasierten Krebserkrankungen geheilt zu haben [15].

In einem Interview aus der Wochenzeitschrift Die Zeit mit der Vorsitzenden des Deutschen Zentralvereins homöopathischer Ärzte, Cornelia Bajic, findet sich folgende zentrale Stelle: „ZEIT: Sie sagen, es sei unseriös, Heilsversprechen abzugeben. Auf Ihrer Homepage steht aber folgende Aussage: ,Ein sorgfältig ausgewähltes homöopathisches Arzneimittel heilt schnell, sanft, sicher, nebenwirkungsfrei und dauerhaft auch schwere, akute und chronische Erkrankungen, wie Migräne, Neurodermitis, Asthma bronchiale, Colitis, Rheumatismus u.v.a., für die sonst nur Linderung, aber keine Heilung möglich ist. Dies gilt auch für akute Krankheiten bakterieller oder viraler Natur.' Das ist ein konkretes Heilsversprechen. Ein Beispiel für das, was Sie gerade als Kennzeichen für unseriöse Anbieter genannt haben. - Bajic: Da haben Sie recht, wahrscheinlich muss ich den Text ändern in ,kann heilen. Homöopathie, genau wie Schulmedizin, kann nicht alles. Das muss man mit dem Patienten besprechen“ [16]. Die Überzeugung vom Nutzen der Homöopathie ist hier offenbar unerschütterlich; „kann heilen“ ist für Kranke etwa mit Migräne oder Colitis ein nicht grundsätzlich abgeschwächtes Heilungsversprechen.
Bundesgesundheitsbl 2020 - 63:541-547 https://doi.org/10.1007/s00103-020-03125-8 (c) Der/die Autor(en) 2020

\section{N. Schmacke}

\section{Homöopathie: Heilslehre „ohne Substanz"}

\section{Zusammenfassung}

Die Homöopathie gehört in Deutschland zu den häufig angewendeten alternativen Heilmethoden. In diesem Diskussionsbeitrag soll erörtert und analysiert werden, warum die Homöopathie nicht zur Medizin gehören sollte und vielmehr als Glaubenskonzept zu verstehen ist, das außerhalb wissenschaftlicher Methoden angesiedelt ist. Erläutert werden die klinischen, rechtlichen und politischen Dimensionen der Homöopathiedebatte. Schließlich wird die Frage der Legitimität von Placeboanwendungen im Lichte der Forderung nach patientenzentrierter Medizin diskutiert.

\section{Schlüsselwörter}

Homöopathie · Heilslehren · Evidenzbasierte Medizin · Placebo · Gesundheitspolitik · Komplementärmedizin · Wissenschaftlichkeit

\section{Homeopathy: insubstantial doctrine of salvation}

\section{Abstract}

Homeopathy is one of the frequently used alternative healing methods in Germany. This article is intended to discuss and analyze why homeopathy should not be part of medicine and should rather be understood as a concept of belief that lies outside of scientific methods. The clinical, legal, and political dimensions of the homeopathy debate are explained. Finally, the question of the legitimacy of placebo applications is discussed in light of the demand for patientcentered medicine.

\section{Keywords}

Homeopathy · Health doctrines - Evidencebased medicine - Placebo - Health politics . Complementary medicine $\cdot$ Scientificity

\section{Subjektiver Nutzen und Placeboeffekte}

Nun berichten aber viele Menschen, dass sie durch die Einnahme von Homöopathika ihre Beschwerden verloren oder sich deutlich besser gefühlt haben. Aus wissenschaftlicher Sicht ist dieser Befund alles andere als erstaunlich. 
Im weiten Feld von weniger bedeutsamen Befindlichkeitsstörungen und Erkrankungssymptomen bessert sich vieles durch Abwarten, egal, ob oder welche Mittel zum Einsatz gekommen sind. In extremen Ausnahmefällen gibt es auch Spontanheilungen schwerer Erkrankungen.

Denkbar ist auch, dass Menschen, die zur Behandlung ihrer Erkrankung die Homöopathie anwenden, parallel auch die "Schulmedizin“ in Anspruch nehmen und die erzielten Behandlungserfolge dann der Homöopathie zuschreiben. Auch bei Erkrankungen mit chronisch rezidivierenden Verläufen scheint es leicht, in der Situation von Remissionen jedweder Behandlungsmethode den Erfolg zuzumessen.

Und schließlich führt diese Thematik zur „Placebodebatte“. So unstrittig es ist, dass die verschiedensten Kontextfaktoren (von der Farbe eines Medikaments über den Arztkittel bis zum räumlichen therapeutischen Ambiente) den Verlauf von Erkrankungen günstig beeinflussen können und dass dabei auch Verfahren wie die homöopathische Erstanamnese oder die Einnahme von Homöopathika eine Bedeutung haben können, so wichtig ist es, daran zu erinnern, dass spürbare therapeutische Fortschritte in der Medizin immer nur erreichbar waren - und künftig sein werden -, wenn eine spezifische Wirksamkeit von Behandlungsverfahren über Placeboeffekte hinaus gegeben ist.

Die Effektstärke von Placebos wird zudem seit jeher drastisch überschätzt [17] und selbst wichtige Vertreter der Placebothese warnen vor derartigen Überschätzungen: Ted Kaptchuk, Professor für Medizin an der US-amerikanischen Harvard Medical School, schrieb: „Of course, placebo effects are modest as compared with the impressive results achieved by lifesaving surgery and powerful, well-targeted medications" [18].

Placebo wird in der zeitgenössischen Forschung aber auch als „unterschätztes“ Therapeutikum propagiert, so beispielsweise von Winfried Rief [19]. Rief fühlt sich durch eine eigene Studie [20] bestätigt, bei der eine Intervention mit dem Ziel einer realistischen Einstimmung von Patienten vor Herzkranzgefäßoperatio- nen auf ihre postoperative Belastbarkeit das Ergebnis zeitigte, dass die Patienten dieses Studienarms in Selbstauskünften über eine höhere Belastbarkeit und Lebensqualität berichteten. Wieso er hieraus die Schlussfolgerung zog, es habe sich um Placeboeffekte gehandelt, ist im Licht der Forschung zur kognitiven Verhaltenstherapie rätselhaft. Zur eigenständigen Bedeutung von Placebo wird auch mit der Studie von Howick et al. [21] argumentiert. Diese Arbeit ist ein Beitrag zur Methodologie der Placeboforschung und die Autoren weisen explizit darauf hin, dass ihre Ergebnisse nicht dafürsprechen, Placebos (etwa in der Schmerzbehandlung) aktiver Therapie gleichzustellen.

Es wird weiter argumentiert, dass das Verordnen von Placebos doch per se nicht unrecht sei. Die Debatte ist komplizierter, als sie auf den ersten Blick erscheint. Hier sei nur darauf verwiesen, dass Placebos - ethisch vertretbar - allenfalls mit dem eindeutigen Hinweis auf das Fehlen von spezifischer Wirksamkeit eingesetzt werden dürfen, dass aber genau dieser Hinweis in der Homöopathie ja nicht gegeben werden kann, damit etwas "geschieht".

Sehr häufig findet sich schließlich das Argument, die „Schulmedizin“ bewirke ungleich mehr unerwünschte Nebenwirkungen, als dies die Homöopathie je tun könne. Aber auch dieses Argument ist nicht einleuchtend, da eine verständige Medizin selbstredend das Verhältnis von erwünschten und unerwünschten Wirkungen immer mit den Patientinnen und Patienten besprechen muss. Wenn dies im Alltag nicht passiert, und dafür gibt es leidvolle Belege, kann das kein Argument für den Einsatz unwirksamer Verfahren wie der Homöopathie sein.

\section{Gründe für die Inanspruch- nahme und Inkonsistenzen in der Behandlung}

Es existiert der übergreifende Eindruck, dass Nutzer der Homöopathie dort vor allem das suchen, was sie in der „Schulmedizin“ vermissen: „Gehört, gesehen und verstanden werden" [22]. Die Forschungslage ist nicht so dicht wie oft vermutet. Häufig genannt wer- den folgende vier Motive für die Inanspruchnahme der Homöopathie [23]: 1.) Empfehlungen aus dem Freundesund Bekanntenkreis, 2.) Überzeugtsein vom Konzept der Individualisierung von Diagnostik und Therapie, 3.) Angst vor Nebenwirkungen der "Allopathie“ (nichthomöopathische Behandlungsmethoden), 4.) Enttäuschungen über fehlende Empathie, unzureichende Akzeptanz individueller Krankheitsdeutungen und Nichternstnehmen wahrgenommener Nebenwirkungen. Es wirkt logisch, dass die ausführliche homöopathische Erstanamnese den hier durchscheinenden Bedürfnissen in besonderem $\mathrm{Maß}$ gerecht wird. Umso erstaunlicher ist, dass es keine systematischen Analysen dieses Herzstücks der Homöopathie gibt. So fehlen vor allem Studien zum Vergleich etwa mit der personenzentrierten Gesprächstherapie nach Rogers [24] oder mit Kurzzeitpsychotherapie.

Es ist aber offensichtlich so, dass sich zwischen bestimmten Kranken und Homöopathinnen wie Homöopathen frühzeitig eine belastbare Passung einstellt, die alle Inkonsistenzen zu überstehen scheint, wie das vielfache Suchen nach dem "richtigen“ Mittel, das Fehlen jedes Ansatzes von partizipativer Entscheidungsfindung, das Verschweigen der Forschungsergebnisse zum ausschließlichen Placeboeffekt der Homöopathika und auch die traditionell nach der ausführlichen Erstanamnese sich anschließenden verkürzten direktiven Beratungen zum Mittelwechsel oder zu Lebensstiländerungen. Aus soziologischer und psychologischer Sicht muss auf einen wesentlichen Befund hingewiesen werden: Während für die Medizin die Ära der partizipativen Entscheidungsfindung und gewissermaßen auch der legitimen Skepsis gegenüber dem Expertentum ausgerufen wurde wie voluntaristisch bzw. unvollendet das auch immer sein mag -, hat die Homöopathie eine klassisch paternalistische Ausrichtung.

\section{Politik und Homöopathie}

Als Folge des Conterganskandals wurde 1978 das deutsche Arzneimittelgesetz erarbeitet, das im weiteren Verlauf in- 
ternationalen Entwicklungen folgend von der pharmazeutischen Industrie ein Stufenkonzept des Nachweises eines vertretbaren Nutzen-Risiko-Profils neuer Arzneimittel verlangte in der entscheidenden Stufe mittels prospektiver randomisierter kontrollierter Studien. Schwächen der Ergebnisse dieser schärferen Gesetzgebung sind immer wieder Gegenstand pharmakologischer und klinischer Debatten gewesen, so vor allem zum Fehlen einer sogenannten vierten Hürde, welche den Herstellern abverlangen würde, das Nutzen-RisikoVerhältnis unter Alltagsbedingungen und über längere Zeiträume zu untersuchen. Das für die Marktzulassung zuständige Bundesinstitut für Arzneimittel und Medizinprodukte (BfArM) akzeptiert im Widerspruch zu dieser wissenschaftsbasierten Zulassungspolitik Homöopathika als Arzneimittel und legt extrem niedrige Hürden für die Marktzulassung zugrunde, nämlich die schlichte Registrierung, bestenfalls die Befürwortung der Zulassung durch eine eigens dafür geschaffene Kommission, die mehrheitlich aus Anhängern der Homöopathie besteht (Binnenkonsens).

Homöopathika erhalten als gesetzlich legitimierte Arzneimittel für alle Laien den ersten Anschein einer der "Allopathie“ gleichzustellenden Arzneimitteltherapie, obwohl wissenschaftliche Wirksamkeitsnachweise fehlen. Der Gesetzgeber hat im Sozialgesetzbuch den Ausnahmetatbestand der „besonderen Therapierichtungen" geschaffen (gemeinsam mit anthroposophischer Medizin und Phytotherapie), die von der Verordnung innerhalb der Gesetzlichen Krankenversicherung nicht ausgeschlossen werden dürfen. Nahezu alle deutschen Krankenkassen erstatten heute, wahrscheinlich aus Wettbewerbsgründen, entweder pauschaliert Zuschüsse zur homöopathischen Behandlung oder übernehmen in sogenannten Selektivverträgen komplette Behandlungsserien [25]. Gesetzgeber und alle bisherigen Regierungsparteien im Bund haben an dieser Sonderregelung für die Homöopathie bisher nicht gerüttelt, wobei dies eher wahltaktischem Kalkül zu entspringen scheint als fachlicher Überzeugung.
Demgegenüber wurde im Vereinigten Königreich [26] und in Frankreich [27] in den letzten Jahren die Homöopathie aus der Erstattungspflicht des Gesundheitswesens entlassen. In der Schweiz [26] ist es geschickter Lobbyarbeit geschuldet, dass die Homöopathie und andere ,alternative" Verfahren von der Sozialversicherung erstattet werden. Grundlage war eine Bürgerbefragung, wissenschaftliche Gründe spielten keine Rolle: Die vorübergehend geforderte Evaluation fand letztendlich nicht statt.

Es sei schließlich darauf hingewiesen, dass ein weiteres Argument pro Homöopathika gern verwendet wird: In einer freien, demokratischen Gesellschaft müssen die Bürgerinnen und Bürger das Recht haben, selber über die Wahl von Behandlungsverfahren zu entscheiden. Das könnte, polemisch formuliert, dazu führen, dass auch Wallfahrten nach Lourdes von den gesetzlichen Krankenkassen finanziert werden müssten: Auch von dort werden konstant eindrucksvolle Behandlungserfolge mitgeteilt. Alle Bürgerinnen und Bürger haben unstrittig das Recht, im Rahmen der Aufklärungspflichten von Behandlern die Verfahren zu wählen, die sie für angemessen halten. Eine solidarische soziale Krankenversicherung aber kann und sollte wirklich nur solche Methoden anerkennen, für die es methodisch hochwertige Nutzenbelege gibt, gleichgültig, ob sie der „Alternativmedizin“ zugerechnet werden oder nicht. Dies nicht zuletzt zum Schutz der Versicherten vor unkalkulierbaren Risiken und einer Belastung des solidarisch finanzierten Gesundheitswesens aus nicht nachvollziehbaren Gründen [28].

\section{Quintessenz}

Wissenschaftsorientierte Medizin basiert unabweisbar auf methodisch belastbaren Nutzenbelegen und auf ausdrücklicher Patientenzentrierung: Die (nicht immer spontan geäußerten) Bedürfnisse von Patientinnen und Patienten müssen integraler Bestandteil von Behandlungskonzepten sein. Persönliche Zuwendung ist ein unverzichtbarer Bestandteil guter Medizin. Dass die erfahrbare Welt der heutigen Gesundheitsversorgung nicht selten gegen beide Prinzipien guter Medizin verstößt, ist so wahr wie kein Argument für den Ersatz wissenschaftsorientierter Medizin durch Heilslehren ohne belastbare Nutzenbelege, die etwa seit den 70er-Jahren des 20. Jahrhunderts im Windschatten der Esoterikbewegung Auftrieb erlebt haben. Die wesentlich ältere Homöopathie konnte ab den 1990erJahren offensichtlich von diesem Trend stärker profitieren.

Homöopathie fußt, wie Heyll [29] analysiert hat, auf der Selbsttäuschung der Therapeutinnen und Therapeuten und der Täuschung der Patientinnen und Patienten. Natürlich sind - Opportunisten ausgenommen - die Homöopathinnen und Homöopathen davon überzeugt, ein wirksames Behandlungskonzept in der Hand zu haben. Trotzdem täuschen sie die Kranken mit der Propagierung des Ähnlichkeits- und des Verdünnungsprinzips.

Das in den letzten Jahren stark in den Vordergrund getretene Argument der Legitimität von Behandlungsverfahren, die nicht mehrals Placeboeffekte auslösen, ist weder aus medizinischer noch aus ethischer Sicht akzeptabel. Selbst wenn Kranken erläutert wird, dass eine Intervention nach wissenschaftlicher Erkenntnis keine spezifische Wirksamkeit beinhaltet, muss man sich fragen, warum positive Kontexteffekte durch Scheintherapie erzeugt werden sollen statt durch Zuhören und ein verlässliches Arbeitsbündnis zwischen Ärztinnen/Ärzten und Patientinnen/Patienten. Vollständig inakzeptabel ist die maßlose Überschätzung der stets nachweisbaren Placeboeffekte: Wenn Homöopathen vorgeben, Krebs, Schlaganfall und jedwede Krankheit tatsächlich heilen oder sie anstelle einer Impfprophylaxe anwenden zu können, handelt es sich um unverantwortliches Belügen von Kranken: das wesentliche Argument gegen die staatliche Toleranz der Homöopathie.

Es ist auch wahr: Immer werden Menschen nach dem Übersinnlichen gerade in verzweifelten Krankheitssituationen suchen, weniger dramatisch auch zur Konstruktion stimmiger Lebenskonzepte. Diese menschlich zu nennende Konstante sollte jedoch kein Teil der Medizin sein. Sie ist eine - tatsächlich relevante 
- Ausdrucksform von Glaubenskonzepten.

Es bleibt noch die Frage, wie die wissenschaftsorientierte Medizin sich gegenüber Kranken verhalten möge, die von der Homöopathie überzeugt sind. Ihnen ist der immer gebotene Respekt vor ihren persönlichen Einschätzungen und Haltungen zu erweisen, verbunden mit dem Angebot, wo immer es von beiden Seiten sinnvoll erscheint, auch wieder einen gemeinsamen Weg auf dem Boden verlässlicher Forschungsergebnisse $\mathrm{zu}$ suchen. Die Suche nach „common ground“ darf nicht davon abgelenkt werden, dass Menschen phasenoder dauerhaft nach Lösungen außerhalb fachlich begründbarer Untersuchungsund Behandlungsverfahren suchen.

\section{Schlussbemerkung}

Menschen können dazu neigen, gerade im Erkrankungsfall magische Lösungen den rationalen vorzuziehen. Diese Neigung begleitete die Menschheit bis in die Ära der heutigen Medizin, die inzwischen handfesten Nutzen vorweisen kann - nicht für alle Erkrankungen, aber in einem Ausmaß, das es in den vorangegangenen Generationen nicht gegeben hat. Es zeigt sich, dass auch die Ärzteschaft vor evidenzfreien Glaubenskonzepten nicht gefeit ist. Dass es aber von der Harvard University [30] bis zur Universitätsklinik München [31] im Establishment der Forschung und Ausbildung Befürworter der Integration von Homöopathie und anderen ähnlich unwirksamen Verfahren gibt, muss nachdenklich stimmen und darf nicht akzeptiert werden. Es erstaunt, welche „Klimmzüge“ unternommen werden, um doch eine spezifische Wirksamkeit der Homöopathie für denkbar zu halten und weitere Forschung zu fordern. Dabei sollte allein der Blick auf die beiden Kernannahmen der Homöopathie (das Ähnlichkeits- und das Verdünnungsprinzip) reichen, um die Analogie zum Märchen „Des Kaisers neue Kleider“ zu ziehen: „Aber er hat ja gar nichts an" [32].

In der Stellungnahme der Arbeitsgemeinschaft der Wissenschaftlich Medizinischen Fachgesellschaften e. V. vom 24.01.2020 zum Arbeitsentwurf der neu- en Approbationsordnung heißt es: „Das Wahlfach ,Homöopathie' sollte auf jeden Fall gestrichen werden, da es dem wissenschaftlichen Charakter des Studiums nicht gerecht wird“ [33]. Die Homöopathie ist in der Tat im doppelten Sinn eine Heilslehre „ohne Substanz“.

\section{Korrespondenzadresse}

Prof. Dr. med. Norbert Schmacke

Bibliothekstrasse 1, 28359 Bremen,

Deutschland

schmacke@uni-bremen.de

Danksagung. Ich danke Frau Dr. med. Natalie Grams für kritische Anmerkungen zum Text.

Funding. Open Access funding provided by Projekt DEAL.

\section{Einhaltung ethischer Richtlinien}

Interessenkonflikt. N. Schmacke gibt an, dass kein Interessenkonflikt besteht.

Für diesen Beitrag wurden von den Autoren keine Studien an Menschen oder Tieren durchgeführt. Für die aufgeführten Studien gelten die jeweils dort angegebenen ethischen Richtlinien.

Open Access. Dieser Artikel wird unter der Creative Commons Namensnennung 4.0 International Lizenz veröffentlicht, welche die Nutzung, Vervielfältigung, Bearbeitung, Verbreitung und Wiedergabe in jeglichem Medium und Format erlaubt, sofern Sie den/die ursprünglichen Autor(en) und die Quelle ordnungsgemäß nennen, einen Link zur Creative Commons Lizenz beifügen und angeben, ob Änderungen vorgenommen wurden.

Die in diesem Artikel enthaltenen Bilder und sonstiges Drittmaterial unterliegen ebenfalls der genannten Creative Commons Lizenz, sofern sich aus der Abbildungslegende nichts anderes ergibt. Sofern das betreffende Material nicht unter der genannten Creative Commons Lizenz steht und die betreffende Handlung nicht nach gesetzlichen Vorschriften erlaubt ist, ist für die oben aufgeführten Weiterverwendungen des Materials die Einwilligung des jeweiligen Rechteinhabers einzuholen.

Weitere Details zur Lizenz entnehmen Sie bitte der Lizenzinformation auf http://creativecommons.org/ licenses/by/4.0/deed.de.

\section{Literatur}

1. Schmacke N (2015) Der Zauber der Homöopathie. Die unheilvolle Beschwörung von Politik und Öffentlichkeit. In:SchmackeN (Hrsg) Der Glaubean die Globuli. Die Verheißungen der Homöopathie. Suhrkamp, Berlin, S80-147

2. Gräfen U (2005) Samuel Hahnemann, ein Mensch voller Widersprüche. https:// www.aerztezeitung.de/panorama/article/ 352061/samuel-hahnemann-mensch-vollerwidersprueche.html.Zugegriffen: 6. Febr. 2020

3. Hood B (2011) Übernatürlich? Natürlich! Warum wir an das Unglaubliche glauben. Spektrum, Heidelberg

4. Weymayr C (2013) Scientabilität - ein Konzept zum Umgang der EbM mit homöopathischen Arzneimitteln. Z Evid Fortbild Qual Gesundhwes 107:606-610

5. Wölfing A (1974) Entstehung und Bedeutung des Begriffs Schulmedizin. Die Auseinandersetzung zwischen der naturwissenschaftlichen Medizin und Vertretern anderer Heilmethoden im 19. Und 20. Jahrhundert. Dissertation, Medizinische Fakultät, Albert-Ludwigs-UniversitätFreiburg i.Br.

6. KappaufH (2020) Was fehlt Ihnen? Woher-Wohin? Mut für eine mitmenschliche Medizin, 3. Aufl. TWENTYSIX, Norderstedt

7. Schmacke N (2015) Der Zauber der Homöopathie. Die unheilvolle Beschwörung von Politik und Öffentlichkeit.In:Schmacke N(Hrsg) Der Glaubean die Globuli. Die Verheißungen der Homöopathie. Suhrkamp, Berlin, S80-147 (87-99)

8. National Health and Medical Research Council (2015) NHMRC information paper: evidence on the effectiveness of homeopathy for treating health conditions. https://www.nhmrc.gov.au/sites/ default/files/images/nhmrc-information-papereffectiveness-of-homeopathy.pdf. Zugegriffen: 6 . Febr. 2020

9. House of Commons (2010) Science and Technology Committee-fourth report. Evidence check 2: homeopathy. https://publications.parliament.uk/ $\mathrm{pa} / \mathrm{cm} 200910 / \mathrm{cmselect} / \mathrm{cmsctech} / 45 / 4502 . \mathrm{htm}$. Zugegriffen:6. Febr. 2020

10. WissHom (2016) Der aktuelle Stand der Forschung zur Homöopathie. http://www.wisshom.de/ dokumente/upload/7cda0_forschungsreader 2016_ergschutzgeb\%C3\%BChr_180713.pd. Zugegriffen: 6. Febr. 2020

11. WissHom (2016) FAQ zur Forschung in der Homöopathie. https://www.homoeopathie-online. info/faq-zur-forschung-in-der-homoeopathie/. Zugegriffen:6. Febr. 2020

12. Informationsnetzwerk Homöopathie (2016) Erklärung des INH zur Veröffentlichung der WissHom: "Der aktuelle Stand der Forschung zur Homöopathie". https:// netzwerk-homoeopathie.info/offizielleerklaerung-des-inh-zur-veroeffentlichung-derwisshom-der-aktuelle-stand-der-forschung-zurhomoeopathie/.Zugegriffen:6. Febr. 2020

13. Mathie RT, Lloyd SM, Legg LA, Clausen J, Moss S, Davidson JR, Ford I (2014) Randomised placebocontrolled trials of individualised homeopathic treatment: systematic review and meta-analysis. Syst Rev 3:142. https://doi.org/10.1186/20464053-3-142

14. Straumann F (2015) «lst das bei Chirurgen so anders?». https://www.tagesanzeiger.ch/ wissen/medizin-und-psychologie/lst-das-beiChirurgen-so-anders/story/17098489. Zugegriffen: 6. Febr. 2020

15. Schmacke N (2015) Der Zauber der Homöopathie. Die unheilvolle Beschwörung von Politik und Öffentlichkeit.In:Schmacke N (Hrsg) Der Glaubean die Globuli. Die Verheißungen der Homöopathie. Suhrkamp, Berlin, S 80-147 (ausführlich hierzu 87-97)

16. Maier J, Schweitzer J (2017) „Homöopathie kann nicht alles". https://www.zeit.de/2017/26/ cornelia-bajic-homoeopathie-aerzte-interview. Zugegriffen:6. Febr. 2020

17. Hróbjartsson A, Gøtzsche PC (2004) Placebo interventions for all clinical conditions. Cochrane Database Syst Rev 3:CD3974 
18. Kaptchuk TJ, Franklin G, Miller FG (2015) Placebo effects in medicine. NEngl J Med 373:8-9

19. Rief W (2018) Placebos als bessere Medizin. https://www.sueddeutsche.de/gesundheit/ homoeopathie-placebos-als-bessere-medizin- 1 . 4099754. Zugegriffen: 6. Febr. 2020

20. Juergens MC, Seekatz B, Moosdorf RG, Petrie KJ, Rief W (2010) Illness belies before cardiac surgery predict disability, quality of life, and depression 3 months later.JPsychosom Res 68:553-560

21. Howick J, Friedemann C, Tsakok M et al (2013) Are treatments more effective than placebos? A systematic review and meta-analysis. PLoS One 8:e62599

22. Schmacke N, Stamer M, Müller V (2014) Gehört, gesehen und verstanden werden: Überlegungen zu den Lehren aus der Homöopathieforschung. ZAllg Med 90:251-255 (insb. S252)

23. Ebd., S. 252

24. Kreuziger A (2000) Carl Rogers: Die Entwicklung der personenzentrierten Gesprächstherapie. https:/www.carlrogers.de/entwicklungpersonenzentrierte-gespraechstherapie.html. Zugegriffen: 6. Febr. 2020

25. Schmacke N (2015) Der Zauber der Homöopathie. Die unheilvolle Beschwörung von Politik und Öffentlichkeit. In:SchmackeN (Hrsg) Der Glaube an die Globuli. Die Verheißungen der Homöopathie. Suhrkamp, Berlin, S 80-147 (zu den arzneimittelund sozialrechtlichen Grundlagen s. S 115-121)

26. Blasius H (2018) Urteil: Homöopathie bleibt von der Erstattung ausgeschlossen. https://www. deutsche-apotheker-zeitung.de/news/artikel/ 2018/06/13/urteil-homoeopathie-bleibt-vonder-erstattung-ausgeschlossen. Zugegriffen: 6 . Febr. 2020

27. Spiegel (2019) Krankenkassen zahlen nicht mehr für Globuli. https://www.spiegel.de/gesundheit/ diagnose/homoepathie-krankenkassen-infrankreich-erstatten-globuli-nicht-mehr-a1276636.html.Zugegriffen: 6. Febr. 2020

28. Schmacke N (2015) Der Zauber der Homöopathie. Die unheilvolle Beschwörung von Politik und Öffentlichkeit. In:Schmacke N (Hrsg) Der Glaubean die Globuli. Die Verheißungen der Homöopathie. Suhrkamp, Berlin, S80-147 (S128ff.)

29. Heyll U (2015) Franz Anton Mesmer, Benjamin Franklin und die Geburt der alternativen Medizin. In: Schmacke N (Hrsg) Der Glaube an die Globuli. Die Verheißungen der Homöopathie. Suhrkamp, Berlin, S 17-79

30. Atwood K (2009) Harvard Medical School: veritas for sale (part III). https://sciencebasedmedicine. org/harvard-medical-school-veritas-for-salepart-iii/.Zugegriffen:6. Febr. 2020

31. http://www.klinikum.uni-muenchen.de/ Kinderklinik-und-Kinderpoliklinik-im-Drvon-Haunerschen-Kinderspital/de/zentren/ perinatalzentrum-im/kreissaal/indes.html Letzter Zugriff:6. Febr. 2020

32. Andersen HC (1862) Des Kaisers neue Kleider. https://maerchen.com/andersen/des-kaisersneue-kleider.php.Zugegriffen:6. Febr. 2020

33. AWMF (2019) Stellungnahmeder Arbeitsgemeinschaft der Wissenschaftlichen Medizinischen Fachgesellschaften (AWMF) zum Arbeitsentwurf des Bundesministeriums für Gesundheit „Approbationsordnung für Ärzte und Ärztinnen (ÄApprO)" vom 29.11.2019. https://www.awmf. org/fileadmin/user_upload/Stellungnahmen/ Aus-_und_Weiterbildung/20200124_AWMF_ Stellungnahme_AEApprO_Final_be.pdf. Zugegriffen: 6. Febr. 2020 